\title{
A versatile electrical impedance calibration laboratory based on a digital impedance bridge
}

\author{
Andreas Elmholdt Christensen ${ }^{1, *}$ \\ ${ }^{1}$ Trescal A/S, Electrical Reference Department, Mads Clausens Vej 12, 8600 Silkeborg, Denmark
}

\begin{abstract}
A four-terminal pair fully digital impedance ratio bridge is described. Its principle of operation including balancing procedure and calculation of unknown impedance value is presented. An initial 1:1 ratio check of the bridge has been performed. Systematic errors of the bridge design and actions to deal with these errors are shown.
\end{abstract}

\section{Introduction}

Calibration of artefact impedance standards such as standard capacitors, standard inductors and AC resistors, (figure 1) has been carried out for decades on the primary level. These calibrations have mainly been performed at well-established National Metrology Institutes (NMIs) in the field of DC resistance and impedance metrology. In the field of electrical measurement there is an ever growing need for precise and accurate capacitance and inductance measurements, that are traceable to the SI.

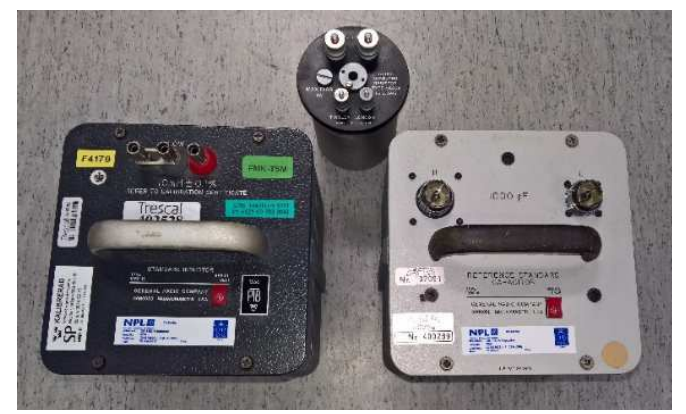

Fig. 1. Impedance standards. Left: General Radio $10 \mathrm{mH}$ Standard Inductor. Centre: Tinsley $100 \mathrm{ohm} \mathrm{AC/DC} \mathrm{Standard}$ Resistor. Right: General Radio 1000 pF Reference Standard Capacitor

Only a limited number of countries world-wide have a primary impedance laboratory, that is capable of realizing the capacitance and inductance scales traceable to the SI with measurement uncertainties at the $10^{-6}$ level or below. The reason for this is that the realisation at this level demand numerous setups, none of which use exclusively off-the-shelf instruments. Design, construction and operation of primary level setups exceed the budget of most laboratories and demand highly skilled manpower $[1,2]$.

Most NMIs or Designated Institutes (DIs) in countries like Denmark have a large set of standard capacitors, standard inductors and standard resistors. These standards are annually shipped to a primary level NMI for calibration, to get traceability to the SI and be able to provide a calibration service for the NMI's or DI's customers. The author's laboratory has like other laboratories experienced unexpected jumps in the values of the standards. Jumps which after some investigation are expected to come from transportation effects such as temperature and humidity fluctuations or mechanical shocks. Due to this and the fact that good quality off-the-shelf digital sources and digitizers are widely available a new generation of digital impedance bridges has seen the light of day.

On a general level there are two types of digital impedance bridges, sourcing bridges (DAC-based) [3] and sampling bridges (ADC-based) [4].

Two European research projects have been dealing with this subject. In 2013 - 2016 a Joint Research Project (JRP) in the framework of "The European Metrology Research Programme" titled "Automated impedance metrology extending the quantum toolbox for electricity" (AIM QuTE) had the goal to extend Josephson impedance bridges capabilities to the whole complex plane with the best level of uncertainties $(0.05 \mathrm{ppm})$ and in parallel to develop fully digital bridges to reduce the operator workload imposed on national metrology institutes for the realisation of the impedances scales and for calibrations [5]. A currently running follow-up JRP in the framework of "The European Metrology Programme for Innovation and Research" titled "A Versatile electrical impedance calibration laboratory based on digital impedance bridges (VersICaL)" has the goal to expand the application of digital impedance bridges among developing NMIs and DIs. The project consortium includes experienced NMIs that have participated in the AIM QuTE and who will assist the less experienced partners to create and operate an in-house realisation of the capacitance and inductance scales based on digital impedance bridges [6].

This paper will present the current state of the Trescal Ltd. Denmark's sampling based digital impedance bridge.

\section{Setup}

* Corresponding author: andreas.christensen@trescal.com 
The Trescal four-terminal pair (4TP) sampling based fully digital impedance ratio bridge consists of off-the-shelf available equipment in addition to a home build coaxial multiplexer (MUX).

Figure 2 shows a schematic diagram and a photograph of the bridge when measuring the ratio of two $12.906 \mathrm{k} \Omega$ resistors.

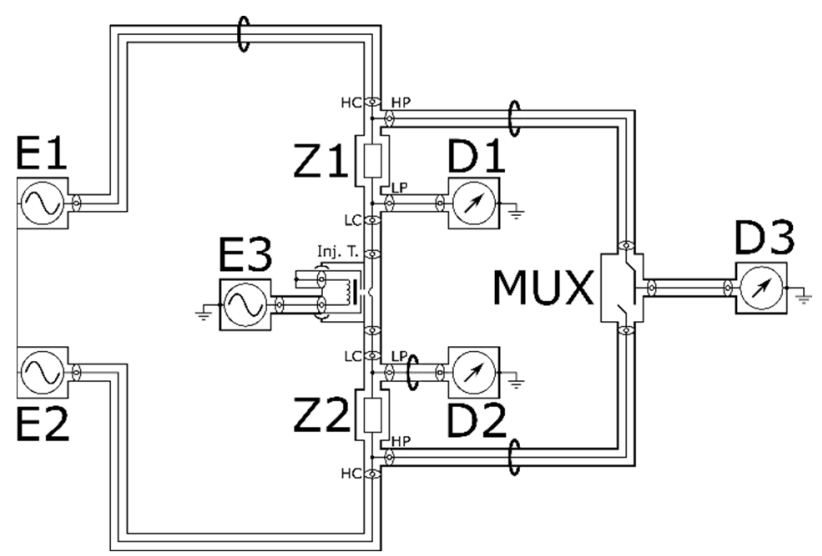

(a)

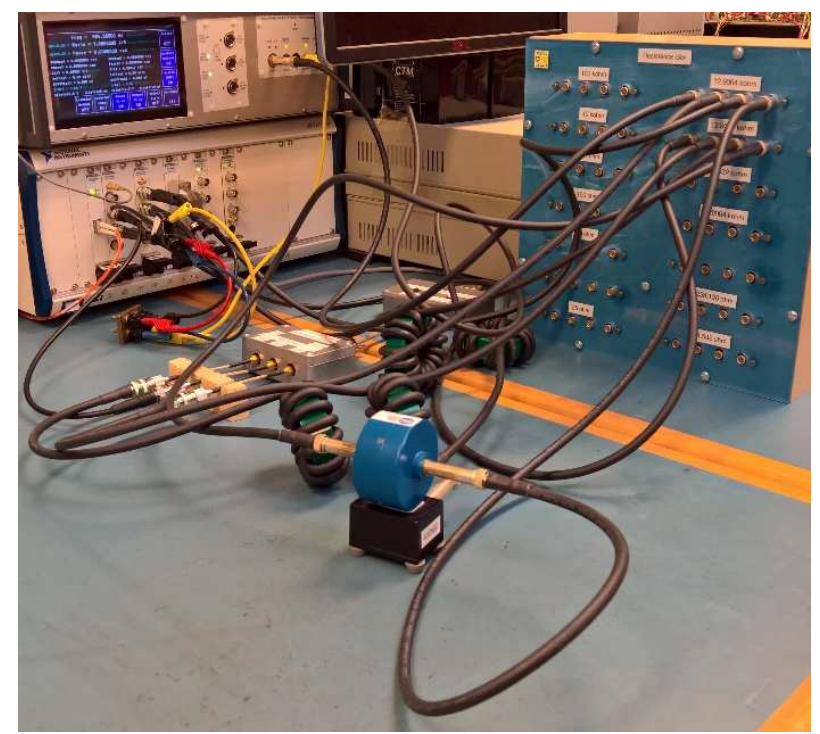

(b)

Fig. 2. Sampling based four-terminal pair fully digital impedance bridge: (a) simplified schematic, (b) photograph of the implemented setup.

A 2-channel digital synthesized source (DSS) by M. Kampik drives the bridge (E1 and E2 of figure 2(a) and upper left corner of figure 2(b)). E1 and E2 are connected to the high current (HC) ports of the two impedances. A NI PXI-4461 drives the injection transformer (E3 and Inj. T. of figure 2(a) and the blue/black device at the bottom of figure 2(b)) and digitizer D3 on the same board measures the high potential (HP) ports of each impedance sequentially by switching the MUX. The MUX is controlled using a NI PXI-2567. A second NI PXI-4461 measures the two low potential (LP) ports of the impedances. To prevent external interference from entering the setup four current equalisers are placed strategically in the setup. These have been drawn as back half circles around the cables in figure 2(a).
This bridge can compare any type of impedances R-R, CC, L-L, R-C, R-L, and C-L and it can be constructed in minutes. It's basically a plug and play solution when having the right software tool for instrument control and data analysis. To be able to compare a 4TP reference AC resistor to a $3 \mathrm{~T}$ inductor or capacitor one needs an adapter. In this case adapter corrections have to be applied to the measurement results [7].

\section{Principle of operation}

A 4TP impedance is defined as [1]

$$
Z_{4 T P}=\frac{V^{H P}}{I^{L C}},
$$

with the defining conditions

$$
\begin{gathered}
I^{H P} \equiv 0, \\
V^{L P} \equiv 0,
\end{gathered}
$$

and

$$
I^{L P} \equiv 0
$$

The software is set to balance the bridge by having E1 fixed and adjusting E2 and E3 until the defining conditions stated above comply for both impedances $\mathrm{Z} 1$ and $\mathrm{Z} 2$.

\subsection{Balancing}

Various types of balancing algorithms have been presented in the literature. After testing several algorithms, it was decided to use the secant method which is one of the simplest ones [8]. Sources and detectors are matched in pairs. D1 is paired with E2 because adjustments of E2 have the most significant effect on D1. E3 is matched with a pseudo detector D4 = D1 $-\mathrm{D} 2$. When D1 is zero and D4 is zero it follows that D2 is zero. E2 and E3 are adjusted sequentially according to the secant method until the root sum of squares of the real and imaginary parts of D1 and D4 is less than $10^{-6}$ of $V_{1}^{H P}$.

\subsection{Final measurement}

When the balancing procedure is complete the complex impedance ratio is equal to the complex voltage ratio of the HP ports of the impedances

$$
\frac{Z_{1}}{Z_{2}}=\frac{V_{1}^{H P}}{V_{2}^{H P}}
$$

The two complex voltages are both measured by the same digitizer in a timespan of less than one second by changing the position of the MUX. Comparing equal impedances will result in equal voltages on the HP ports on the impedances, $V_{1}^{H P}$ and $V_{2}^{H P}$. Any offset or gain error of D3 will in this case cancel out. 
In reality, one voltage ratio comes from two measurements. First a 'forward' measurement is performed where the reference is positioned at $\mathrm{Z} 1$ and the device under test (DUT) is positioned at Z2. This is followed by a second measurement 'reverse' where the reference is positioned at $\mathrm{Z} 2$ and the DUT is positioned at $\mathrm{Z1}$. This is done to cancel out any inequality in the measurement setup. The result is the average of the forward and the reverse measurement.

Knowing the resistance value at a given frequency and the time constant of a reference $\mathrm{AC}$ resistor one can calculate the AC resistance, capacitance or inductance of an unknown device [7].

\section{Initial check}

An initial check of the new bridge has been performed by comparing two $12.906 \mathrm{k} \Omega$ AC reference resistors which have been calibrated at METAS in Switzerland. The certificate values are $\mathrm{R} 1=12.90617 \mathrm{k} \Omega$ and $\mathrm{R} 2=$ $12.90631 \mathrm{k} \Omega$ respectively. Figure 2(b) shows the setup of this measurement.

$\mathrm{R} 1$ was set to be the reference of this comparison. The value of R2 in the forward configuration was measured to be $12.906310 \mathrm{k} \Omega$ and $12.906313 \mathrm{k} \Omega$ in the reverse configuration. The average value of R2 was 12.906312 $\mathrm{k} \Omega$ which was satisfactory for this test

\section{Systematic errors and actions}

The setup is subject to two significant systematic errors. When comparing non-equal impedances the linearity error of the main detector, D3, directly affects the impedance ratio. A method to determine the error of the digitizer and correct for it on a sufficient level of accuracy has been presented for high frequency impedance bridges [9]. A series-parallel capacitive device has been constructed to measure the D3 linearity error, but is yet to be tested, see figure 3 .
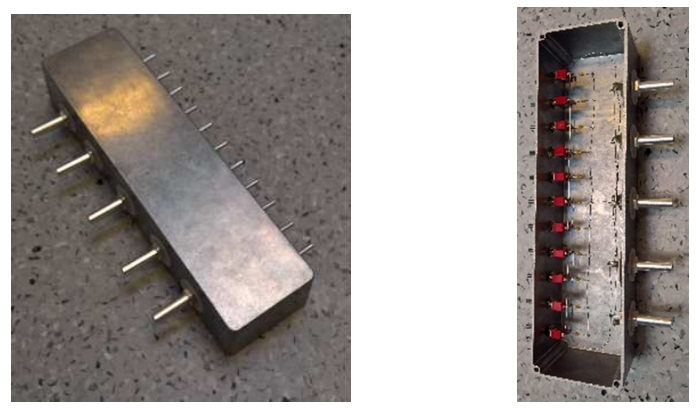

Fig. 3. Trescal design of series-parallel capacitance device.

The second systematic error occurs at high impedances e.g. $1000 \mathrm{pF}$ which has a reactance of $1.6 \mathrm{M} \Omega$ at $100 \mathrm{~Hz}$. The input impedance of the main digitizer in differential configuration is $1 \mathrm{M} \Omega \| 217 \mathrm{pF}$. In this case the 4TP defining condition given in equation (2) is no longer fulfilled, as current will flow through the digitizer rather than the impedance itself. At the time of writing a buffer amplifier having an input impedance of $400 \mathrm{G} \Omega$ is being added to the output of the MUX. This will ensure that the defining condition in equation (2) is fulfilled at all times. The result of this modification is yet to be tested.

\section{Conclusion}

The new Trescal four-terminal pair (4TP) sampling based on a fully digital impedance ratio bridge has been presented.

Initial tests have shown that point of concept is working well for a 1:1 comparison. Systematic errors have been identified. Ideas to correct for the systematic errors have been presented, but are yet to be tested.

\section{References}

1. S. Awan, B. Kibble, J. Schurr, "Coaxial Electrical Circuits for Interference-Free Measurements", (2011), ISBN: 978-1-84919-069-5

2. L. Callegaro, "Electrical Impedance", (2013), ISBN: 978-1-4398-4910-1

3. J. Kučera, J. Kováč, "A Reconfigurable Four Terminal-Pair Digitally Assisted and Fully Digital Impedance Ratio Bridge", IEEE Transactions on Instrumentation and Measurement, 67,5, pp. 11991206, (2018), doi: 10.1109/TIM.2018.2790538

4. F. Overney, B. Jeanneret, "RLC Bridge Based on an Automated Synchronous Sampling System", IEEE Transactions on Instrumentation and Measurement, 60,7, pp. 2393-2398, (2011), doi: 10.1109/TIM.2010.2100650

5. https://www.ptb.de/emrp/sib53-home.html

6. https://sites.google.com/inrim.it/versical

7. F. Overney, B. Jeanneret, "Realization of an inductance scale traceable to the quantum Hall effect using an automated synchronous sampling system", Metrologia, 47,6, pp. 690-698, (2010), doi: 10.1088/0026-1394/47/6/008

8. L. Callegaro, "On strategies for automatic bridge balancing”, IEEE Transactions on Instrumentation and Measurement, 54,2, pp. 529-532, (2005), doi: 10.1109/TIM.2004.843126

9. J. Kučera, R. Sedláček, J. Boháček, “A new capacitance device for calibration of $\mathrm{N}: 1 \mathrm{HF}$ inductive voltage dividers", CPEM 2010 Conf. Digest, pp. 390-391, (2010), doi: 10.1109/CPEM.2010.5544205

Acknowledgment: This project has received funding from the EMPIR programme co-financed by Participating States and from the European Union's Horizon 2020 research and innovation programme.
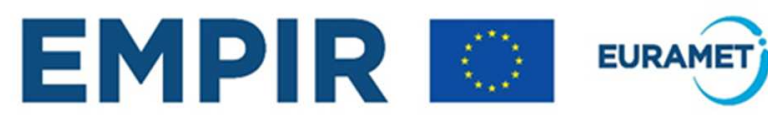

The EMPIR initiative is co-funded by the European Union's Horizon 2020 research and innovation programme and the EMPIR Participating States 